\title{
CLOBAZAM-A NEW HYPNOTIC?
}

\author{
C.M. KESSON, J.M.B. GRAY \& D.H. LAWSON \\ Clinical Pharmacology Service, Glasgow Royal Infirmary, Glasgow G4 OSF
}

1 A double-blind randomized trial to compare the relative efficacy of the 1,5-benzodiazepine, clobazam, the 1,4-benzodiazepine nitrazepam, and placebo as hypnotics was carried out.

2 A preference technique was used and the analyses were performed sequentially.

3 The results confirmed that nitrazepam was superior to placebo as a hypnotic but failed to show that clobazam was superior to either placebo or nitrazepam in a ratio of $2: 1$. Thus, it seems likely that the hypnotic properties of clobazam are minimal.

4 Clobazam is not suitable for use as a hypnotic in hospitalized patients.

\section{Introduction}

Recently benzodiazepines have superceded barbiturates as hypnotics of choice because of their greater efficacy and safety (Greenblatt \& Shader, 1974; Barraclough, 1974).

Clobazam is a new benzodiazepine differing from other compounds in this group of psychotherapeutic agents because the nitrogen atoms in the heterocyclic ring are in the 1,5-position instead of the 1,4-position and the characteristic azomethinic function is lacking (Barzaghi, Fournex \& Mantegazza, 1973).

Previous studies on human performance after hypnotic ingestion have shown that with 1,4-benzodiazepines taken at night, performance may be impaired the following day (Borland \& Nicholson, 1975). Different drugs of the benzodiazepine group have different effects on performance, and whereas diazepam, which has been shown to lead to diminished performance in man (Linnoila \& Mattila, 1973), causes a decrease in reaction time, it has been demonstrated recently that the 1,5-benzodiazepine, produces no alteration in human reaction time (Borland \& Nicholson, 1974). Indeed this drug is reported to have limited effects on human performance of skilled tasks (Berry, Burtless, Grubb \& Hoare, 1974). Clobazam, however, has been reported to be a more active sedative than chlordiazepoxide hydrochloride in animals (Barzaghi et al., 1973). The present study was designed to test the efficacy of this 1,5-benzodiazepine as a hypnotic in man.

\section{Methods}

A double-blind randomized trial was set up to compare the relative efficacy of clobazam $(20 \mathrm{mg})$, nitrazepam $(5 \mathrm{mg})$ and placebo, the methods used being similar to those previously described (Thomson, 1958; Jick, Sloan \& Dinan, 1966; Kesson, Gray \& Lawson, 1976). The patients studied were those consecutive admissions to medical wards who were conscious, able to communicate a preference to the investigators, and not suffering primarily from hepatic or respiratory insufficiency. None had been previously taking hypnotics on a regular basis. All gave their informed consent.

The drugs were administered in identical capsules in random order. After two hypnotic-free nights in hospital, each patient received one of the two comparison drugs on night 3 and the other on night 4. On the fifth day their preference was recorded and a record kept of any adverse reactions. In each comparison group a limit of 30 preferences was set. Analyses were performed sequentially (Jick et al., 1966; Kesson et al., 1976; Snell \& Armitage, 1957; Parsons \& Thomson, 1961). Preference lines were drawn such that line A (Figure 1) was crossed when the ratio of preference of drug A over drug B was 2:1 and line $B$ crossed when the preference ratio was 1:1. Line $\mathbf{C}$ was crossed if neither preference line could be crossed within the total number of patients available when the error limits were set at $5 \%$.

\section{Results}

In all, 161 patients were admitted to the various patient preference studies, of whom 53\% were male. Their mean age was 56 years, mean admission blood urea was $6 \mathrm{mmol} l \mathrm{l}$ and mean haematocrit $40 \%$. 


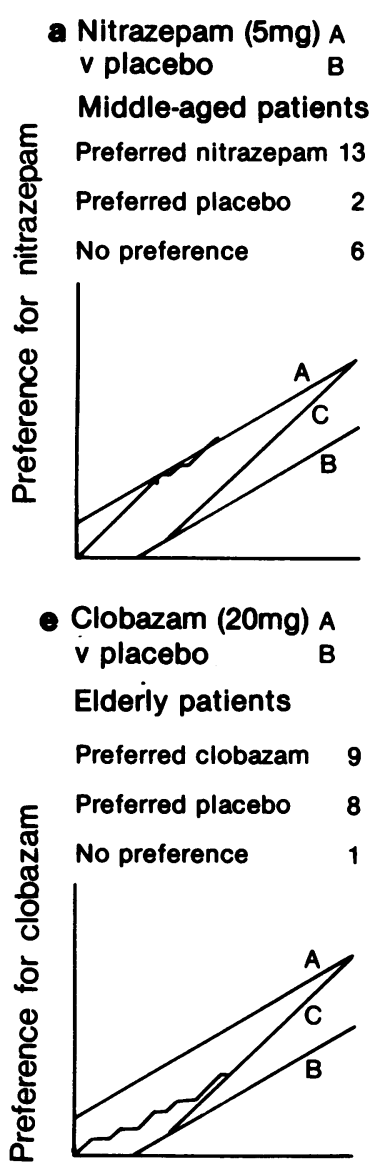
b Nitrazepam (5mg) A $v$ placebo Elderly patients

Preferred nitrazepam 10

Preferred placebo 0

No preference 3

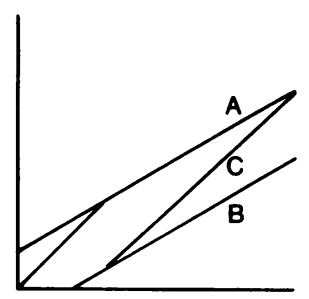

f Clobazam (20mg) A $v$ nitrazepam $(5 \mathrm{mg})$ B Young patients

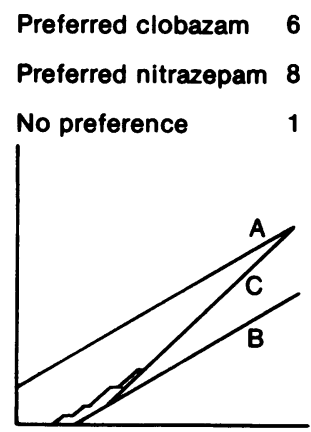

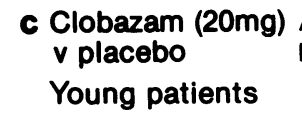

d Clobazam (20mg) A $\checkmark$ placebo B

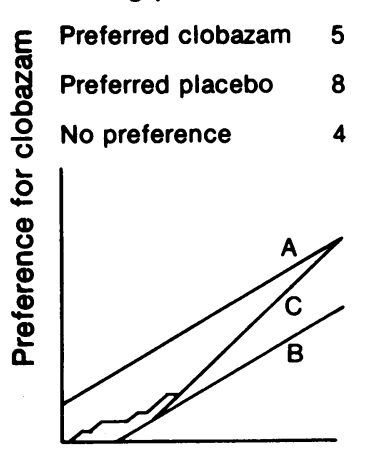

g Clobazam (20mg) A $\checkmark$ nitrazepam $(5 \mathrm{mg})$ B Middle-aged patients

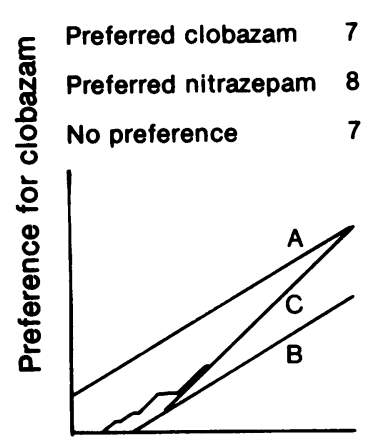
Middle aged patients

Preferred clobazam 13

Preferred placebo 7

No preference $\quad 10$

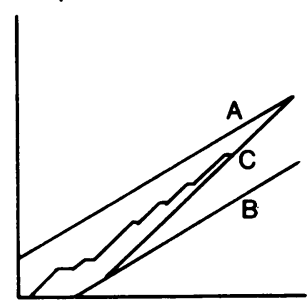

h Clobazam (20mg) A $v$ nitrazepam $(5 \mathrm{mg})$ B Elderly patients

Preferred clobazam 1 Preferred nitrazepam 7 No preference 4

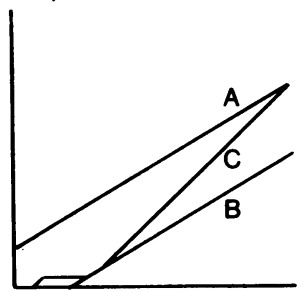

Total preferences

Figure 1 Preferences for the various treatments in the eight studies. For explanation of A, B and C see Mothods.

Study I Nitrazepam (5 mg) v placebo: Middle-aged patients

In this comparison 21 patients participated. Their ages ranged from 46-65 years with a mean age of 58 . Eight were male and 13 female. Six patients could not state a preference for either drug, having slept equally well on both occasions. Of the remainder, 13 preferred nitrazepam and 2 preferred placebo. Thus, the study showed a statistically significant result at the $5 \%$ level in favour of nitrazepam in a $2: 1$ ratio over placebo after 21 patients had been entered (Figure 1a).

Study II Nitrazepam (5 mg) v placebo: Elderly patients

Nineteen patients with an age range of 66-80 years and a mean age of 70 years participated in this study. Six patients could not complete the trial for administrative reasons leaving 8 males and 5 females in the study. Of these, three stated no preference and 10 preferred nitrazepam resulting in a statistically significant result at the $5 \%$ level in favour of nitrazepam in a 2:1 ratio over placebo (Figure 1b).

Study III Clobazam (20 mg) v placebo: Young patients

This comparison involved 18 patients with an age range of 25-45 years and a mean age of 38 years. There were 10 males and eight females. Four patients could not state a preference for either drug, and one was discharged before the trial was completed. Of the remainder, five preferred clozabam and eight preferred placebo. Therefore, the study was terminated as line $\mathbf{C}$ was crossed, indicating a failure to show superiority of clobazam in a 2:1 ratio over placebo within the study limits (Figure 1c). 


\section{Study IV Clobazam (20 mg) v placebo: Middle-aged patients}

This study involved 33 patients with an age range of 46-65 years, and a mean age of 55 years. Twentyfour patients were male and nine were female. Ten were unable to state a preference for either drug. The study was terminated when line $\mathbf{C}$ was crossed, indicating that, within the set limits of the trial, no superiority of one drug over the other was established (Figure 1d).

\section{Study V Clobazam (20 mg) v placebo: Elderly patients}

This comparison involved 19 patients of age range 66-79 years. Their mean age was 70 years, six being male and 13 female. One patient could not state a preference for either drug, and one patient was transferred to another ward before the study was completed. Of the remainder nine preferred clobazam and eight placebo, indicating a failure to show superiority of one drug over the other, within the study limits (Figure 1e).

\section{Study VI Clobazam (20 mg) v nitrazepam (5 mg): Young patients}

This comparison involved 15 patients whose ages ranged from 17-44 years, with a mean age of 31 years. Five were male and 10 female. One patient could not state a preference for either drug. Of the remaining patients, six preferred clobazam and eight nitrazepam (Figure 1f). Thus, this study failed to show either drug as superior to the other at the prescribed doses.

Study VII Clobazam (20 mg) v nitrazepam (5 mg): Middle-aged patients

Twenty-two patients participated in this trial. Their ages ranged from 46-65 years with a mean of 54 years. Thirteen were male and nine female. Seven patients were unable to state a preference for either drug, seven preferred clobazam and eight preferred nitrazepam. This study again failed to show significant superiority of either drug at the prescribed doses (Figure 1g).

\section{References}

BARRACLOUGH, B.M. (1974). Are there safer hypnotics than barbiturates? Lancet, i, 57.

BARZAGHI, F., FOURNEX, R. \& MANTEGAZZA, P.(1973).
Study VIII Clobazam $(20 \mathrm{mg})$ v nitrazepam $(5 \mathrm{mg})$ : Elderly patients

Fourteen patients took part in this study. Their mean age was 72 years (range of 66-77 years). Eleven patients were male and three female. Four patients stated no preference for either drug, and two discontinued the trial for administrative reasons. One patient preferred clobazam and nitrazepam. Thus, the study showed a preference ratio of $1: 1$ between the two drugs (Figure 1h).

\section{Discussion}

The methods used in this study have been shown to be both reliable and reproducible in assessing symptomatic drug therapy qualitatively provided that the basic symptoms under treatment do not vary on a daily basis (Thomson, 1958). The study design was selected because of simplicity, economy of staff time, minimum patient interference, use of each patient as his own control, and the benefits of a sequential analysis design. Zelvelder (1971) in an extensive review of the techniques of evaluating hypnotics, recommended this approach as the most efficient one available for evaluating short-term hypnotic efficacy.

Separate studies were undertaken in young, middleaged and elderly subjects because of previous reports of differences in responsiveness to hypnotics and in particular to benzodiazepines according to age (Kesson et al., 1976; Greenblatt, Allan \& Shader, 1977). The studies have clearly demonstrated that nitrazepam is superior to placebo as a hypnotic. However, the studies failed to demonstrate a clear preference for clobazam over placebo and for clobazam over nitrazepam within the limits of the selected preference ration. It is likely, therefore, that whereas clobazam has a mild hypnotic effect in man, its potency lies somewhere between nitrazepam and placebo. Since there is clearly no need for a hypnotic with this low potency, further studies at lower preference ratios (say $1.5: 1$ ) were not deemed necessary to establish the actual potency of the drug. Nor for the same reasons, was it felt necessary to test the effect of increasing the dose of clobazam. It is concluded that clobazam is not suitable for use as a hypnotic in hospitalized patients.

The authors are grateful to Hoechst Pharmaceuticals Limited for providing a supply of clobazam to carry out this study.
Pharmacological and toxicological properties of clobazam, a new psychotherapeutic agent. ArzneimForsh. (Drug Res.), 23, 683-686. 
BERRY, P.A., BURTLESS, R., GRUBB, D.J. \& HOARE, Margaret V. (1974). An elevation of the effects of clobazam on human motor co-ordination, mental activity and mood. Br. J. clin. Pharmac., 1, 346P.

BORLAND, R.G. \& NICHOLSON, A.N. (1974). Immediate effects on Human Performance of a 1,5-benzodiazepine (clobazam) compared with the 1,4-benzodiazepines, chlordiazepoxide hydrochloride and diazepam. Br. J. clin. Pharmac., 1, 215-221.

BORLAND, R.G. \& NICHOLSON, A.N. (1975). Comparison of the residual effects of two benzodiazepines on human performance. Br. J. clin. Pharmac., 2, 177P.

GREENBLATT, D.J. \& SHADER, R.I. (1974). Benzodiazepines in Clinical Practice. New York: Raven Press.

GREENBLATT, D.J., ALLEN, M.D. SHADER, R.I. (1977). Toxicity of high-dose flurazepam in the elderly. Clin. Pharmac. Ther., 21, 355-361.

JICK, H., SLOAN, D. DINAN, B. (1966). Evaluation of drug efficacy by a preference technique. New Engl. J. Med., 275, 1399-1403.
KESSON, C.M., GRAY, J.M.B. \& LAWSON, D.H. (1976). Benzodiazepine drugs in general medical patients. $B r$. med.J., 1, 680-682.

LINNOILA, M. \& MATTILA, M.J. (1973). Drug interaction on psychomotor skills related to driving: diazepam and alcohol. Eur. J. clin. Pharmac., 5, 186-194.

PARSONS, T.W. THOMSON, T.J. (1961). Methaqualone as a hypnotic. Br. med.J., 1, 171.

SNELL, E.S. \& ARMITAGE, P. (1957). Clinical comparison of diamorphine and pholcodine as cough suppressants. Lancet, i, 860-862.

THOMSON, T.J. (1958). Clinical comparison of methylprylone and quinalbarbitone as hypnotics. $B r$. med. J., 2, 1140-1141.

ZELVELDER, W.G. (1971). Therapeutic evaluation of hypnotics. London: Heinemann.

(Recetved August 25, 1977) 